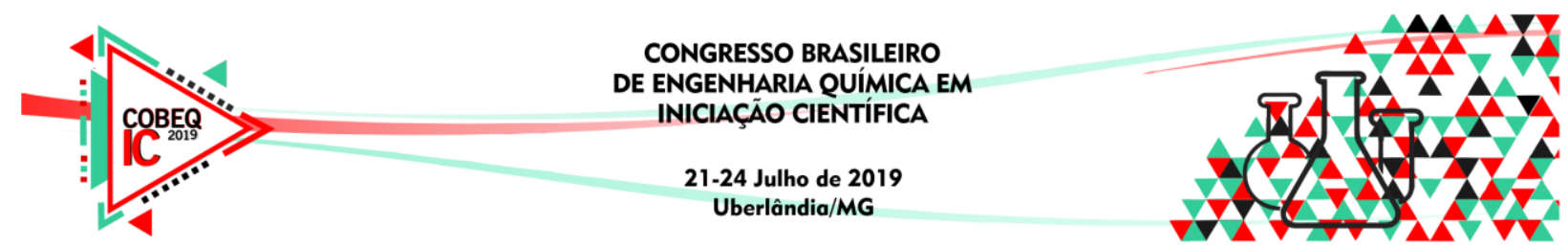

\title{
ESTUDO CINÉTICO DA DECOMPOSIÇÃO TÉRMICA DA COCAÍNA BASE
}

\author{
H. G. MOREIRA ${ }^{1}$, T. F. ZYS ${ }^{2}$, D. I. PELUFO ${ }^{1}$ e A. B. SIQUEIRA ${ }^{2}$ \\ ${ }^{1}$ Universidade Federal de Mato Grosso, Faculdade de Engenharia \\ ${ }^{2}$ Universidade Federal de Mato Grosso, Departamento de Química/ICET \\ E-mail para contato: moreirahitalo@gmail.com
}

\begin{abstract}
RESUMO - A cocaína é uma substância psicoativa extraída das folhas de coca. Muitas técnicas têm sido utilizadas para identificação dessa substância, devido ao seu uso proibitivo no Brasil. Assim, neste trabalho utilizou-se a espectroscopia na região do infravermelho médio (FTIR) e a termogravimetria-análise térmica diferencial (TG-DTA) para caracterizar uma amostra de cocaína e realizar o estudo cinético de seu comportamento térmico. Com as curvas TG-DTA foi possível observar duas etapas de decomposição térmica, porém sendo observado três picos na curva DTA, com formação de uma pequena quantidade de resíduo. Com o FTIR, pode-se determinar que a amostra era de cocaína base e possuía um adulterante. $\mathrm{O}$ estudo cinético da decomposição térmica sugeriu a presença de reações paralelas na decomposição da cocaína, evidenciando a presença de adulterante. Com isso, observa-se que as técnicas utilizadas foram eficientes na identificação e caracterização da amostra de cocaína.
\end{abstract}

\section{INTRODUÇÃO}

A cocaína é um alcaloide, com efeitos psicoativos, extraído das folhas de coca (Erytroxylum coca/novogranetense). Essa substância, de uso proibitivo no Brasil, apresenta duas formas, a base livre, e sal de cocaína, geralmente na forma de cloridrato de cocaína. A molécula de cocaína possuí um anel aromático, dois grupos de ésteres, um grupo alifático de sete carbonos e um grupo amina. A forma de cloridrato de cocaína possuí um átomo de hidrogênio ligado ao grupo amina, devido a presença do cloro (ZACCA et al., 2014).

A identificação dessas duas apresentações da cocaína pode ser realizada através da utilização de diversas técnicas. O Scientific Working Group for the Analysis of Seized Drugs ( $S W G D R U G$ ) é um órgão que instrui sobre as técnicas que podem ser utilizadas na perícia criminal, sendo que o padrão mínimo é a utilização de uma técnica da categoria A e outra de qualquer categoria. Quando não for possível utilizar uma técnica de categoria A, aplicam-se três técnicas de identificação com duas pertencendo a categoria B. Na categoria A estão incluídas técnicas como FTIR, espectroscopia de massa e espectroscopia de Raman. Na categoria B cromatografia gasosa, cromatografia em camada delgada, eletroforese capilar, entre outras. E na categoria $\mathrm{C}$, encontra-se a espectroscopia de fluorescência e ultravioleta, testes colorimétricos, testes de ponto de fusão e imunoensaios (SWGDRUG, 2014).

Essas técnicas de análises são de extrema importância para identificar a presença desse composto, visto que a legislação brasileira prevê que basta presença desse entorpecente em 


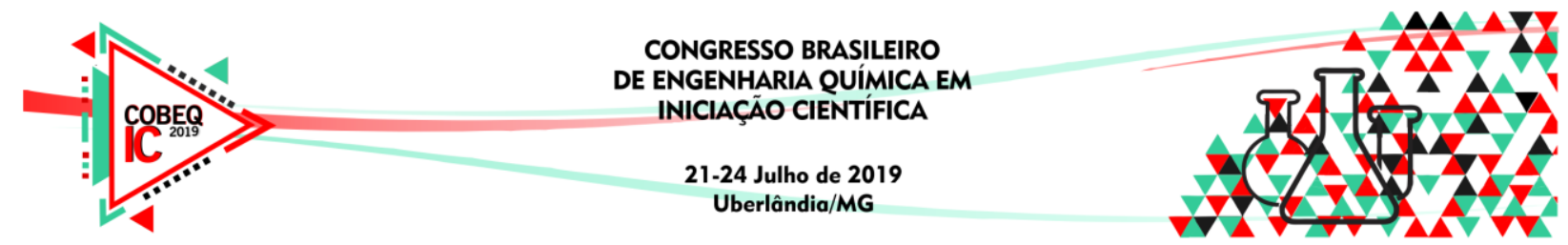

uma amostra apreendida para haver julgamento criminal (BRASIL, 2006). A associação de técnicas quantitativas a essas técnicas já utilizadas apresenta-se como uma forma para obtenção do perfil químico desse tipo de composto, o que auxilia no combate ao tráfico de drogas.

A análise térmica é uma técnica analítica que tem potencial para fornecer diversos dados sobre uma amostra de cocaína, como, por exemplo, etapas de decomposição térmica, temperatura em que ocorre mudança de estado físico, presença de adulterantes e/ou diluentes (IONASHIRO et al., 2014). Outra ferramenta importante é o estudo da cinética de decomposição térmica dos materiais, que fornece informações como a energia de ativação aparente da cocaína.

Assim, este trabalho tem como objetivo realizar o estudo cinético sobre a decomposição térmica da cocaína, aplicando o método isoconvensional sugerido por Flynn-Wall-Ozawa (FWO). Além disso, caracterizar a amostra através das técnicas FTIR e TG-DTA.

\section{MATERIAIS E MÉTODOS}

\subsection{Método de FWO}

Um dos métodos empregados para o estudo da cinética de decomposição térmica de compostos é o método cinético não-isotérmico de FWO, o qual se baseia na utilização da Equação 1 (WENDLANT, 1985). O estudo foi realizado no intervalo de temperatura de $184{ }^{\circ} \mathrm{C}$ à $281{ }^{\circ} \mathrm{C}$.

$$
\log \beta=\log \left(\frac{\mathrm{AE}}{\mathrm{Rg}(\alpha)}\right)-2,315-0,4567 \frac{\mathrm{E}_{\mathrm{a}}}{\mathrm{RT}}
$$

Na Equação 1, os termos $\beta, \mathrm{A}, \mathrm{Ea}, \mathrm{R}$ e T representam a razão de aquecimento, fator de frequência, energia de ativação, constante dos gases ideais e temperatura, respectivamente. $O$ termo $\mathrm{g}(\alpha)$ é uma função característica da reação, sendo que $\alpha$ representa o avanço da reação.

A partir da Equação 1, mantendo $\alpha$ constante, é possível plotar curvas de isoconversão $1 /$ Txlog $(\beta)$. O gráfico gerado possuíra diversas retas paralelas, em que a inclinação das retas é proporcional a $-0,4567 \mathrm{Ea} / \mathrm{R}$. Com isso, pode-se determina a energia de ativação aparente da amostra em estudo (WENDLANT, 1986). O método de FWO foi avaliado nos valores de $\alpha$ de 0,$1 ; 0,2 ; 0,3 ; 0,4 ; 0,5 ; 0,6 ; 0,7 ; 0,8$ e 0,9 .

\subsection{Espectro de infravermelho}

O espectro na região do infravermelho médio foi obtido com emprego do Espectrofotômetro da Nicolet, modelo iS10, com resolução $4 \mathrm{~cm}^{-1}$, na região compreendida entre $4000-600 \mathrm{~cm}^{-1}$, utilizando a técnica de reflectância total atenuada (ATR).

\subsection{Curvas TG-DTA}

As curvas TG-DTA foram obtidas em equipamento DTG-60H, Simultaneous TG-DTA APPARATUS da SHIMADZU. As amostras foram colocadas em cadinho de platina $(70 \mu \mathrm{L})$, com massa de amostra de aproximadamente $7,5 \mathrm{mg}$. Foram realizadas análises nas razões de 


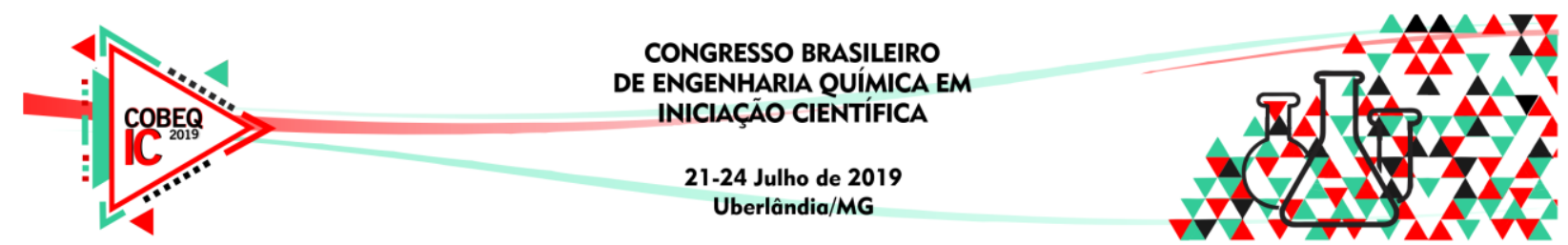

aquecimento de 5, 10, 20, 30 e $40{ }^{\circ} \mathrm{C} \mathrm{min}{ }^{-1}$, atmosfera de ar seco com vazão de $100 \mathrm{~mL} \mathrm{~min}{ }^{-1}$ e intervalo de temperatura de $20-1000^{\circ} \mathrm{C}$.

\section{RESULTADOS E DISCUSSÕES}

\subsection{FTIR}

O FTIR se apresenta como uma análise que permite identificar qual o tipo de cocaína com qual está se trabalhando. A Figura 1 apresenta o FTIR da amostra de cocaína utilizada no estudo cinético. Bandas de intensidade médias presentes em 2976 e $2945 \mathrm{~cm}^{-1}$ indicam que a amostra é de cocaína base e são atribuídas aos estiramentos $\mathrm{C}-\mathrm{H}$ da amostra. Além disso, em $1734 \mathrm{e} 1706 \mathrm{~cm}^{-1}$ observam-se as bandas referentes ao estiramento (v) das duas carbonilas presentes na cocaína, e em 1273, 1226 e $1013 \mathrm{~cm}^{-1}$ os estiramentos do grupo C-O. As bandas em 1038 e $1068 \mathrm{~cm}^{-1}$ são atribuídas às vibrações do anel aromático e a banda $712 \mathrm{~cm}^{-1}$ referente à deformação $(\delta)$ da ligação C-H (RODRIGUES et al., 2013).

Figura 1 - Espectro FTIR da amostra de cocaína.

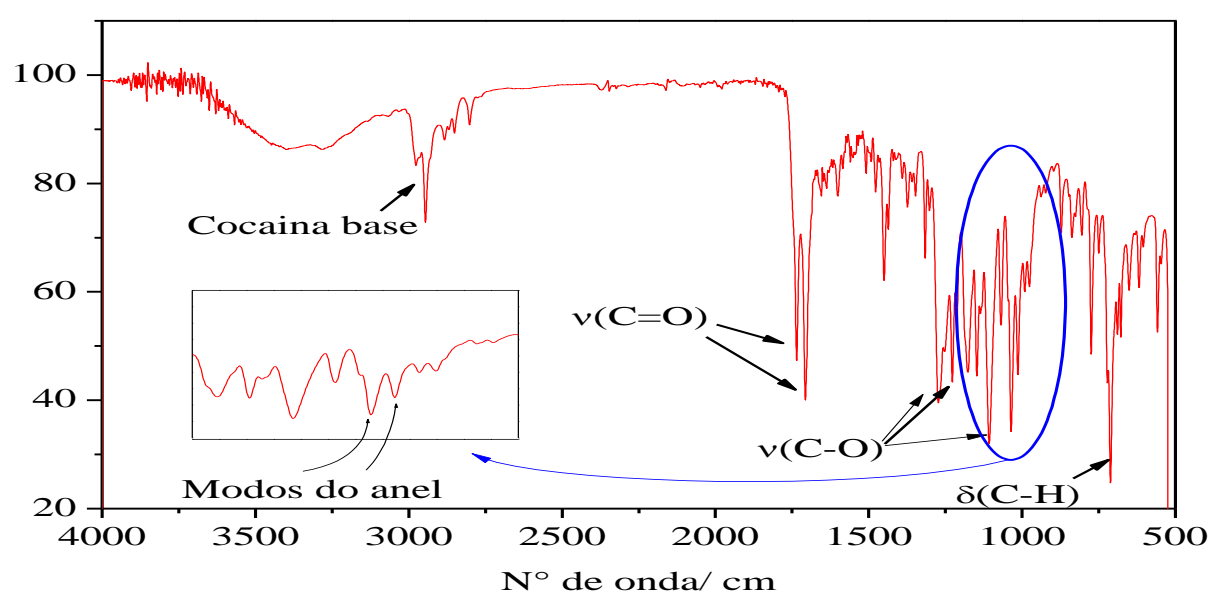

Podem-se observar no FTIR várias bandas que não são da cocaína. Isso indica a presença de outras substâncias na amostra, podendo ser de resíduos do processo de purificação ou adulterantes e/ou diluentes. Algumas dessas bandas sugerem a presença de fenacetina. Segundo Kahmann et al. (2018), as bandas em 1508, 923 e $836 \mathrm{~cm}^{-1}$ podem ser usadas para indicar a presença de fenacetina. Essas bandas são atribuídas, respectivamente, ao estiramento $\mathrm{C}-\mathrm{N}$ e as vibrações $\mathrm{C}-\mathrm{H}$ do anel aromático.

\subsection{TG-DTA}

O perfil de decomposição térmica da cocaína pode ser observado através as curvas TGDTA, fornecendo o número de etapas de composição térmica e informações sobre a estabilidade térmica. As curvas $\mathrm{TG}$ da amostra de cocaína em diferentes razões de aquecimento são apresentadas na Figura 2, em destaque a curva TG-DTA da amostra com $\beta=20{ }^{\circ} \mathrm{C} \mathrm{min}^{-1}$. É possível observar duas etapas de decomposição térmica, com três picos indicados na DTA. O primeiro pico endotérmico, observado em $90^{\circ} \mathrm{C}$, é referente à fusão da cocaína. No segundo evento térmico é observado um pico endotérmico em $227^{\circ} \mathrm{C}$, que é atribuído à evaporação da cocaína. Este evento ocorre com perda de massa de $91,22 \%(\mathrm{~m} / \mathrm{m})$. 


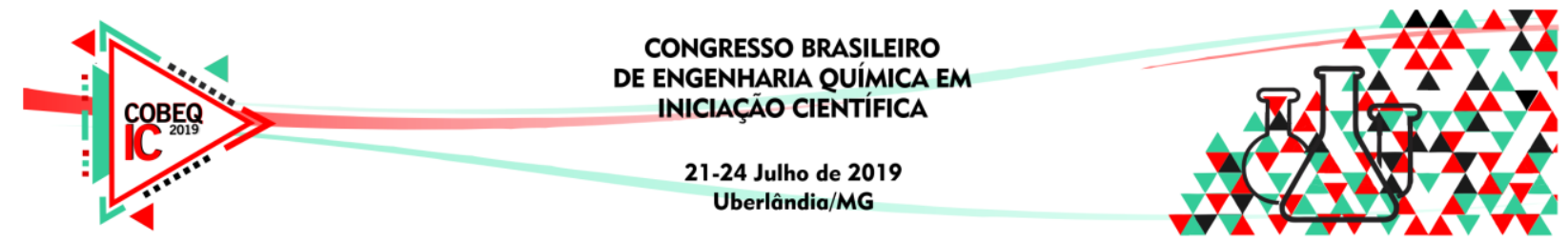

$\mathrm{O}$ terceiro evento térmico, com pico exotérmico em $522^{\circ} \mathrm{C}$ e perda de massa igual a $6,69 \%$ $(\mathrm{m} / \mathrm{m})$, é atribuído à presença de adulterante e/ou diluente na amostra, sugerindo pelo perfil da curva TG-DTA ser amido (CARDOSO et al., 2018).

Figura 2 - Curvas TG da amostra de cocaína em diferentes $\beta$ e curva TG-DTA da amostra $\operatorname{com} \beta=20^{\circ} \mathrm{C} \min ^{-1}$.

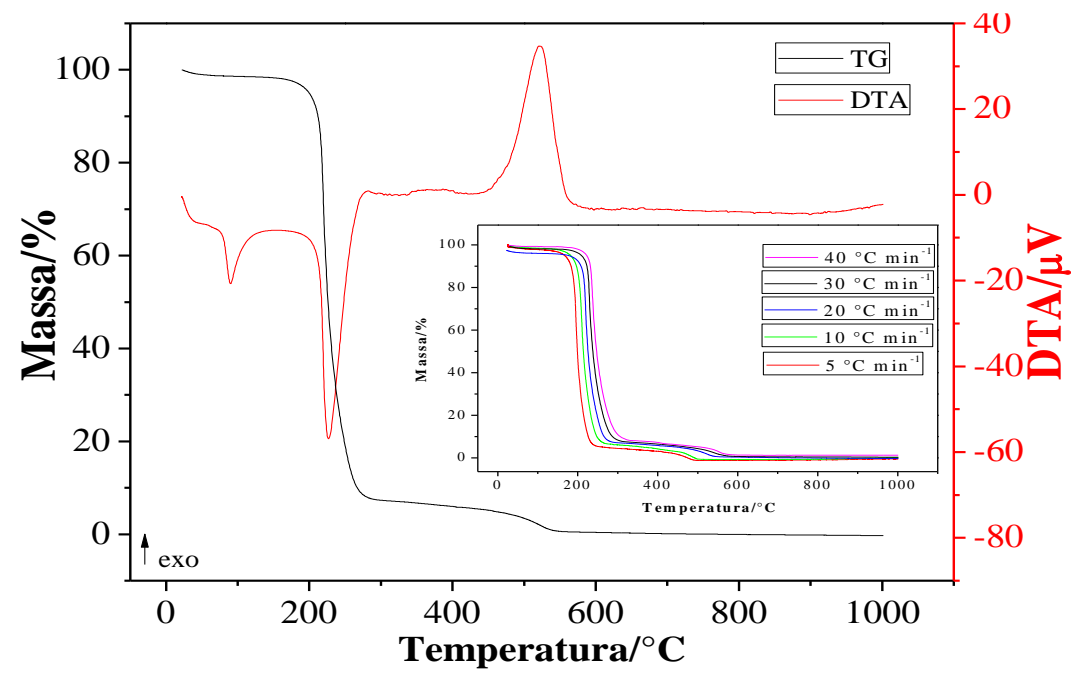

A perda de massa total da amostra foi de $97,91 \%(\mathrm{~m} / \mathrm{m})$. Além disso, apresentou estabilidade térmica até $165^{\circ} \mathrm{C}$. As outras razões de aquecimento apresentaram perfis similares sendo distintas apenas as respectivas temperaturas dos processos térmicos envolvidos, conforme indicado na literatura (IONASHIRO et al., 2014).

O perfil de decomposição térmica da fenacetina, adulterante identificado na amostra de cocaína, está apresentado na Figura 3. Observa-se na curva DTA um pico de fusão em $142{ }^{\circ} \mathrm{C}$ e um pico de evaporação em $279^{\circ} \mathrm{C}$, com perda de massa de $99,44 \%(\mathrm{~m} / \mathrm{m})$.

Figura 3 - Curva TG-DTA da fenacetina a $\beta=20^{\circ} \mathrm{C} \mathrm{min}^{-1}$.

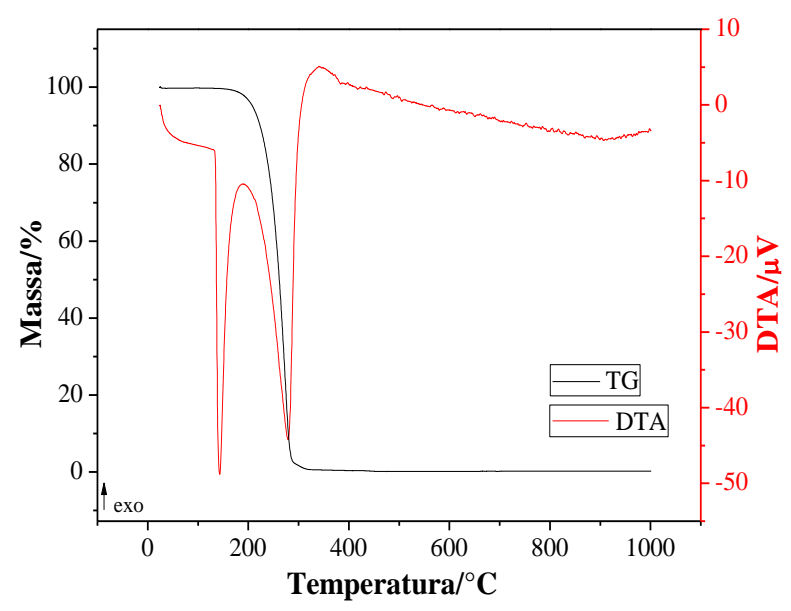

Observa-se que o pico endotérmico na DTA que indica a evaporação da fenacetina (Figura 3) em $279^{\circ} \mathrm{C}$ é muito próximo ao pico endotérmico na DTA que indica a evaporação da amostra de cocaína (Figura 2) em $227^{\circ} \mathrm{C}$. Com isso, o evento térmico de evaporação da cocaína termina antes da evaporação da fenacetina. 


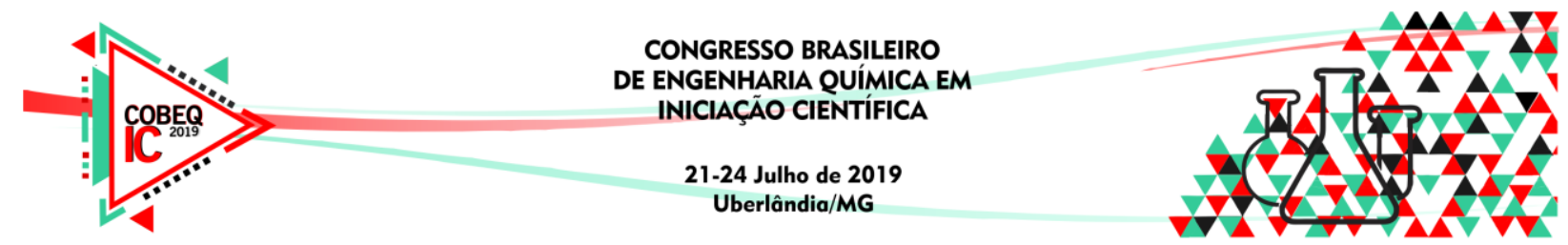

\subsection{Estudo Cinético}

A Figura 4 apresenta as curvas de isoconversão do material utilizando o método não-isotérmico de FWO. O gráfico possibilitou a determinação dos valores de Ea em diferentes graus de conversão, conforme indicado na Figura 5.

Figura 4 - Método FWO para a cocaína: $1 / \operatorname{Tx} \log (\beta)$.

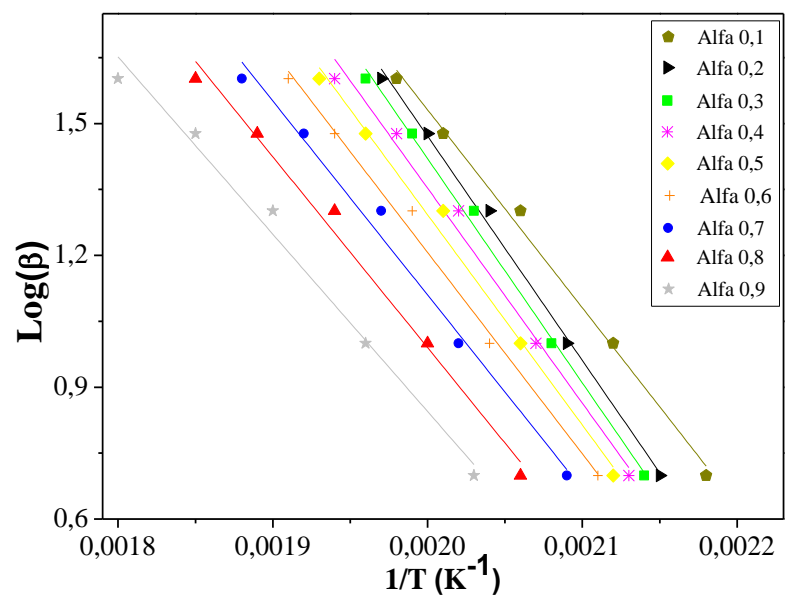

Figura 5 - Gráfico da dependência da Ea com $\alpha$.

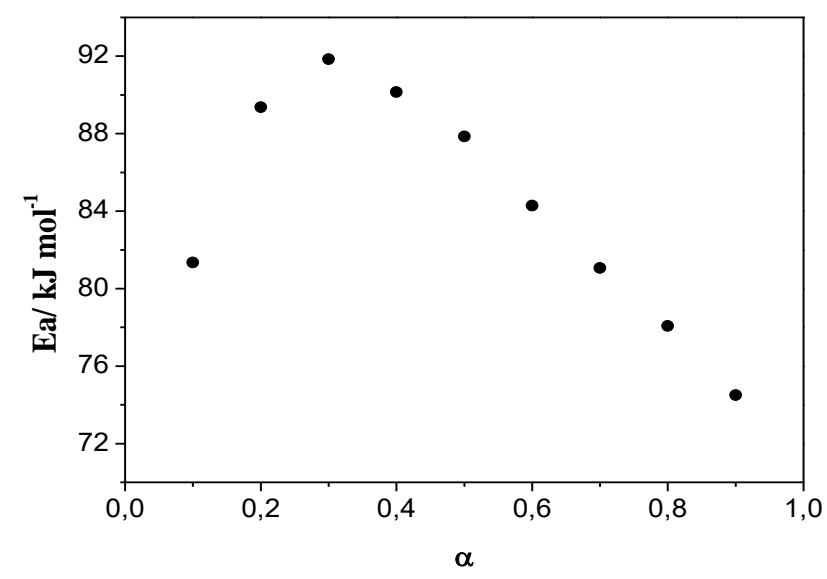

O método não-isotérmico de FWO apresentou uma boa correlação com os dados obtidos, pois foram observados valores de aproximadamente 0,99. A Ea da amostra de cocaína teve uma variação entra os valores de 74,506 e $91,847 \mathrm{~kJ} \mathrm{~mol}^{-1}$. O valor médio da Ea é de $84,276 \mathrm{~kJ} \mathrm{~mol}^{-1}$, com um desvio padrão de $\pm 5,611$.

Pode-se notar através da Figura 5 que a energia de ativação tende a crescer com o aumento de $\alpha$, até aproximadamente $\alpha=0,3$. Em seguida, começa a decrescer com o avanço de $\alpha$. Esse comportamento pode indicar que há reações paralelas durante a evaporação da cocaína. Além disso, segundo Vyazovkin (2015) a energia de ativação para a cinética de fase sólida depende das propriedades do meio. As interações que ocorrem entre moléculas de cocaína e interações cocaína-fenacetina-amido podem levar a variações na energia de ativação do tipo que foram observadas. 


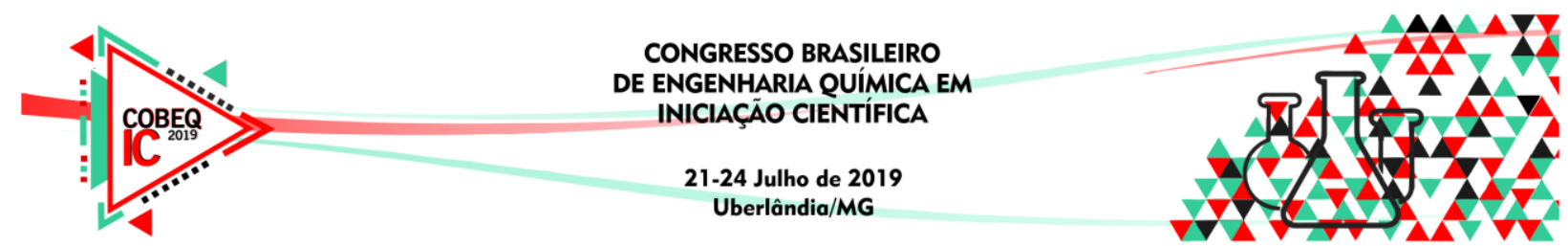

\section{CONCLUSÕES}

A partir das curvas TG-DTA foi possível observar as etapas de decomposição térmica e determinar a presença de adulterantes na amostra. $\mathrm{O}$ estudo cinético da cocaína possibilitou observar processos paralelos que acontecem durante a evaporação da cocaína devido à presença da fenacetina, o qual tem seus eventos decomposição térmica em temperaturas próximas à da cocaína, sugerindo inclusive a formação de um eutético. A presença desse adulterante foi confirmada pelo FTIR. Sendo assim, as técnicas aplicadas foram eficazes na detecção e determinação de adulterantes e/ou diluentes na amostra de cocaína, tendo potencial para aplicações de identificação.

Agradecimentos: CNPq, UFMT e a Shimadzu na pessoa do senhor Antônio Marcos C. D. Beltrão, por ceder o software de cinética para a realização deste trabalho.

\section{REFERÊNCIAS}

BRASIL. Lei $\mathrm{n}^{\circ}$ 11.343, de 23 de agosto de 2006. Instituí sobre o Sistema Nacional de Políticas Pública sobre Drogas. Disponível em: <http://www.planalto.gov.br/ccivil_03/_ato2004-2006/2006/lei/111343.htm>. Acesso em: 24 jan. 2019.

CARDOSO, M. Y.; ZYS, T. F. ; SIQUEIRA, A. B. . Potencialidades da Análise Térmica no Rastreamento de Drogas no Mato Grosso-Brasil. In: XI Congresso Brasileiro de Análise Térmica e Calorimetria, 2018, Rio de Janeiro. Livro de Resumos XI CBRATEC. Rio de Janeiro, 2018.

KAHMANN, A.; ANZANEllo, M. J.; FOGLIATTO, F. S.; MARCELO, M. C. A.; FERRÃO, M. F.; ORTIZ, R. S.; MARIOTTI, K. C. Wavenumber selection method to determine the concentration of cocaine and adulterants in cocaine samples. Journal of Pharmaceutical and Biomedical Analysis, 152, 120-12, 2018. Doi:10.1016/j.jpba.2018.01.050.

IONASHIRO, M.; CAIRES, F. J.; GOMES, J. C. G. Giolito: Fundamentos da termogravimetria e análise térmica diferencial/calorimetria exploratória diferencial. $2^{\mathrm{a}}$ ed.; Vésper: São Paulo, 2014.

RODRIGUES, N. V. S.; CARDOSO, E. M.; ANDRADE, M. V. O.; DONNICI, C. L.; SENA, M. M. Analysis of Seized Cocaine Samples by using Chemometric Methods and FTIR. Spectroscopy. Journal of the Brazilian Chemical Society, 24(3), 507-517, 2013. Doi:10.5935/0103-5053.20130066.

SWGDRUG. Recomendations. Versão 7.0: SWGDRUG, 2014.

ZACCA, J. J. et al. Brazilian Federal Police drug chemical profiling - The PeQui Project. Science \& Justice, 54(4), 300-306, 2014. Disponível em: <http://dx.doi.org/10.1016/j.scijus.2014.02.008>.

WENDLANT, W. WM. Chemical Analysis: Thermal analysis. 3. ed. JOHN WILEY \& SONS: Texas, 1985.

VYAZOVKIN, S. Isoconversional Kinetics of Thermally Stimulated Processes. 1. ed. Springer International Publishing: 2015. 